\title{
Multiagent System In-network Processing In Wireless Sensor Network
}

\author{
Seno Adi Putra ${ }^{1,2}$, Bambang RiyantoTrilaksono ${ }^{1}$, Agung Harsoyo ${ }^{1}$, \\ and Achmad Imam Kistijantoro \\ ${ }^{1}$ School of Electrical Engineering and Informatics, Institut Teknologi Bandung, Indonesia \\ ${ }^{2}$ School of Industrial and System Engineering, Telkom University, Indonesia
}

\begin{abstract}
Wireless Sensor Network (WSN) consists of embedded devices attaching sensor and microelectronic technology, computational capability, and distributed processing. Due to computing capability, it is interesting to implement in-network processing in WSN. It means that data preprocessing is conducted in network to reduce amount of data and processing time in server. One of in-network processing approach is multiagent-based signal processing. Here, Agents deployed in sensor nodes, including mobile agents, make collaboration for data aggregation and preprocessing. This paper describes our proposed method how multiagent system is implemented in real WSN. The method lists the process how global network information is identified by agents in sensor nodes, mobile agent migration plan is conducted by the intelligent agent in head clusters, and the mobile agent migrates hop by hop and performs collaboration with sensor node agents for data preprocessing. We propose vibration of a concrete beam as the case study. Our simulation and experimental results show that the proposed multiagent system works properly.
\end{abstract}

Index Terms: wireless sensor network, multiagent system, and in-network processing.

\section{Introduction}

Wireless sensor network usually contains three main entities: a set of sink nodes, a set of cluster heads $(\mathrm{CH})$, and a set of sensor nodes coordinated by cluster head. A sink node takes into account in processing data collected from sensor nodes, a cluster head is a high computing power sensor node, or can be a single board computer, that coordinates sensor nodes, and a sensor node senses an environmental variable and delivers it to cluster head or sink node. Due to microprocessor deployment in sink node, cluster head, and a sensor node, it is necessary to exploit computing capability in WSN. This capability shifts data processing approach from centralized to distributed-processing, called in-network processing. It allows computational processes to be performed in a sensor node. Sensor nodes should not only send or execute a specific program, but also actively be involved in choosing a decision how to operate network and maintain its resources intelligently. An interesting network operation considering energy resources and leveraging computing capability in a sensor node is multiagent system approach. In this approach, agents make cooperation to reach their goals such as efficient data collection or preprocessing and long life time. Here, agents are deployed in sensor nodes, sink nodes, and cluster heads.

Agent is computer system with capability to perform autonomous action in an environment for specific objectives. It performs perceives, deliberations, and actions to its environment. Intelligent agents commonly have three main behaviors: reactive, proactive, and social. Reactive means agents maintain its interaction with its environment and response to events. Proactive means agents attempt to reach their objectives, not only are encouraged by events, but actively reorganize its network. Social capability is ability of the agent to make cooperation and coordination with others. It is interesting to implement such concepts in wireless sensor network for the case of in-network processing.

Why multiagent system? Today, traditional WSN causes a new problem in managing a large amount of data in a server in which data does not always represent a significant event. An event triggers each sensor node to collect and transmit data to a sink node [1]. In addition, sensor nodes that always transmit data will consume a lot of energy. Thus, the autonomous system that 
leverages intelligent sensing must be considered. The promising approach that leverages autonomous and intelligent system is the multiagent system, which also leverages the concept of mobile agent to overcome problems in bandwidth and energy consumption.

Four types of agent architecture in WSN are described in [2], which defines the architecture of one sensor node per perceptor, the architecture of one agent per sensor node, the architecture of mobile agent, and the architecture of hybrid. First architecture considers sensor node as an individual perceptor coordinated by one agent in sink node or cluster head. This architecture implements centralized agent architecture. In second one, an agent is deployed in each sensor node. This approach implements a distributed processing conducted by autonomous agents. In third one, an agent migrates hop by hop to sensor nodes for data collection or preprocessing. The last one combines one sensor node as preceptor and the mobile agent architecture. In this work, we implement the last architecture of multiagent, which is not described in [2].

The multiagent architecture in WSN is also introduced in [3], which delegates the role of agents into seven definitions, such as an intelligent sensor, a data centric sensor, an autonomy agent, a mobile agent, a directory facilitator, a requester agent, and a provider agent. Another interesting model of multiagent architecture was proposed in [4], called a multiagent hybrid. This model defines many agents in each sensor node, such as a manager resource agent, a capturing agent, a coordinator agent, a task agent, and a mobile agent. However, these two articles neither describe the implementation of their architecture in real sensor node and the mechanism how they works together in the architecture. The employment of multiagent system in WSN was also described in [5]. However, it does not describe agent behaviors and how agents make cooperation. Thus, we need to implement the concept of multiagent system both in simulation environment and in real WSN.

In this work, we also propose the mobile agent, which considers the migration plan. Migration plan means determining an efficient mobile agent route. Migration plan is described in [1], which explains two main issues that have to be overcome by agents located in sink node/cluster head and mobile agent autonomously such as selecting a list of sensor nodes that a mobile agent must visit and an order of visited sensor nodes considering efficient energy consumption, total sensor node, and importance level of information. A migration plan technique for a mobile agent in WSN is described in [6] that proposes algorithm of local closest first, global closest first, near-optimal itinerary, and tree-based itinerary. Delay scalability, potential route efficiency, and traffic load balancing was issued in [7], which compares such algorithms with genetic algorithm considering a correlation network density among energy cost, task duration, and energy-delay time combination. The mobile agent migration plan was also described in [8], which proposes the itinerary energy minimum for first-source-selection algorithm and the itinerary energy minimum algorithm. Other algorithms that can be considered for routing in WSN, but not specific to mobile agent migration, include reinforcement learning, swarm intelligence, evolutionary algorithm, and neural network [9].

After considering architecture design, it is necessary to model agent behavior. A beliefdesire-intention (BDI) and Markov Decision Process (MDP) model described in [10], although not specific to WSN, can be implemented in the agent of sensor node. Another agent behavior is explained in [11] that proposes three main issues, such as a resource management, a communication protocol, and a collaboration schema. It does not only model the individual agent, but also the behavior of agents that exchange information such as a service request, a service report, a state, regulation policy, and BDI. In addition, the goal of agent organization must be considered in application-specific. The organizational goals include responsibility in allocating their own resource, autonomy in task selection, and optimizing parameters that support organization to achieve global optimization such as a long life WSN [12].

The organization objective that must be achieved by agents is energy efficiency for a long life WSN. The concept of strategy-based communication defined in [13] will be considered. It defines aspects that should be considered, such as information importance communication, which reduce communication among sensor nodes, inter-sensor information exchange, and data concatenation. Another strategy that can be consider is compromising two situations, such as 
keeping sensor nodes awake to get high probability in capturing an important information and maintaining the battery level of sensor node by optimizing sleeping strategy without ignoring possibility to capture an important change in its environment [14].

The main contribution of this paper is proposing the multiagent system, which leverages the mobile agent, for supporting in-network processing in real WSN. It combines concepts related to the agent and WSN. Here, we propose three process sequences of in-network processing such as sensor node condition identification conducted by the intelligent agent resided in cluster head, mobile agent migration plan performed by cluster head, and the sensor node agent and the mobile agent collaboration for data aggregation and preprocessing. This paper is divided into four sections. Section 1 is an introduction, Section 2 explains multiagent system for in-network processing in WSN, Section 3 describes experimental results, and Section 4 is a conclusion.

\section{The Proposed Multiagent System In-Network Processing}

In this section, we describe our proposed procedures of in-network processing method. These procedures include identification of network information about sensor nodes condition, mobile agent migration plan, and data aggregation and preprocessing. Acceleration data of concrete beam vibration is the case study. The proposed WSN is shown in Figure 1.

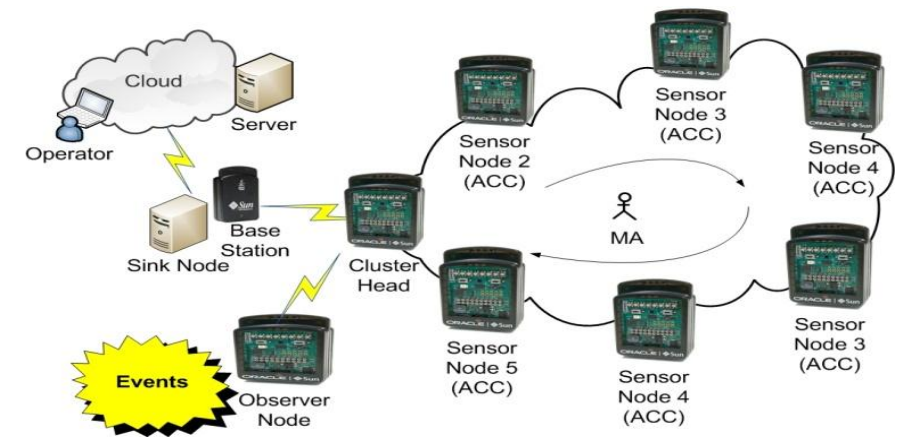

Figure 1. Single cluster of WSN implementing mobile agent (MA)

In our architecture, we define four entities: cluster heads, sensor nodes (ACC) coordinated by a cluster head, an observer node, and a sink node. The cluster head agent is deployed in cluster head and the sensor node agent is deployed in each sensor node. Each sensor node has a mobile agent that can migrate hop by hop to other sensor nodes. The cluster head agent is responsible to identify global network condition and perform migration plan. The sensor node agent is responsible to perform data processing. In our case study, it senses acceleration of concrete beam vibration, and process Fast Fourier Transform (FFT). The mobile agent make cooperation with sensor node agents to aggregate data and transport data from the first to last sensor node, then sends the data to the cluster head. Finally, each cluster head sends the data to the sink node. Innetwork processing will be executed when there is a significant event that hits the concrete beam. Here, the observer node attaching another intelligent agent takes into account for identifying events. For example, in the case of bridge structural health monitoring the observer node is powerful node that detects heavy trucks affecting to significant bridge vibration. This node then sends a message to sensor nodes coordinated by cluster head in order to change their mode from sleep to wake up. For energy conservation, it is necessary to make nodes in sleep mode if no significant event occurs.

\section{A. Identification of Global Network Information}

This section describes the first mechanism how the cluster head agent identifies sensor nodes condition under its coordination after receiving instruction commands of in-network processing from the observer node agent. Sensor node placed in a cluster must register themselves both to their cluster head and neighbor sensor nodes after receiving REG message from the cluster head. 
It is conducted by the sensor node agent that sends a broadcast message to their neighbor sensor nodes and a unicast message to the cluster head. This registration includes sending information about their signal strength (p) and remaining energy level (e). Cluster head and sensor node agent will identify active neighbor sensor nodes. Sensor nodes with low remaining energy level cannot register themselves and unable to join in-network processing. The Cluster head agent then sends broadcast SENSE message to command all agents in each sensor node to start rating calculation of their neighbor sensor nodes. The calculation result produces data structure shown in Figure 2.
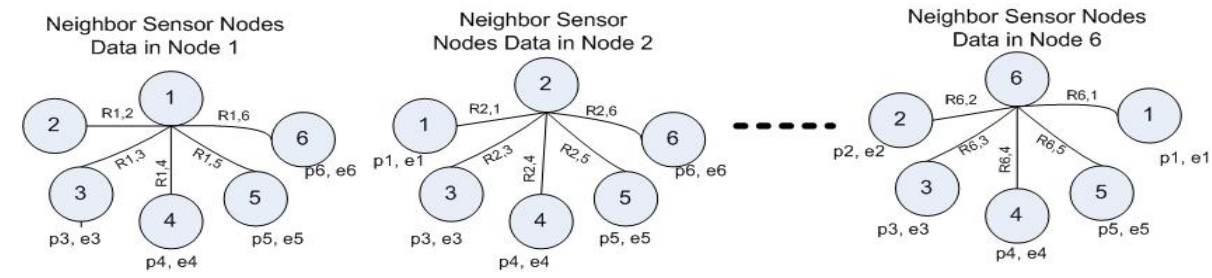

Figure 2. Data structure related to rating of neighbor sensor node condition.

The calculation results of sensor nodes rating are then sent to a cluster head. These data are collected in cluster head forming two-dimensional matrix as shown in Equation 1.

$$
R(p, e)=\left[\begin{array}{cccc}
r_{11} & r_{12} & \ldots & r_{1 N} \\
r_{21} & r_{22} & \ldots & r_{2 N} \\
\ldots & \ldots & \ldots & \ldots \\
r_{N 1} & r_{N 2} & \ldots & r_{N N}
\end{array}\right]
$$

Using this matrix, the cluster head agent starts to process mobile agent migration plan.

\section{B. Mobile Agent Migration Plan}

In this work, we propose two optimization objectives that will be considered by the cluster head agent when performing mobile agent migration plan. These objectives are the signal strength (p) between two sensor nodes and a remaining energy level in each sensor node (e). We call this optimization function as rating of sensor node shown in Equation 2.

$$
\max R(p, e)=\sum_{i=1}^{N} \sum_{j=1}^{N} a_{i j}\left(w_{p} p_{i j}+w_{e} e_{i j}\right)
$$

where $\mathrm{a}_{\mathrm{ij}}=1$ if $\mathrm{i}^{\text {th }}$ and $\mathrm{j}^{\text {th }}$ sensor node are a part of mobile agent route and $\mathrm{a}_{\mathrm{ij}}=0$ if $\mathrm{i}^{\text {th }}$ and $\mathrm{j}^{\text {th }}$ sensor node are not a part of route. Notation $\mathrm{w}_{\mathrm{p}}$ and $\mathrm{w}_{\mathrm{e}}$ are weight of signal strength and energy level respectively. Notation $\mathrm{p}_{\mathrm{ij}}$ and $\mathrm{e}_{\mathrm{ij}}$ are signal strength and energy level between $\mathrm{i}^{\text {th }}$ and $\mathrm{j}^{\text {th }}$ sensor node respectively. The constraint of above objective function includes: the mobile agent is dispatched from a sensor node to another sensor node, a sensor node is only visited from another sensor node, and the mobile agent cannot visit from $i^{\text {th }}$ to $\mathrm{j}^{\text {th }}$ sensor node and go back from $\mathrm{j}^{\text {th }}$ to $\mathrm{i}^{\text {th }}$ sensor node. In this work, we utilize two well-established algorithms: genetic algorithm (population-based) and simulated annealing (single-solution). These two algorithms are processed by the cluster head agent.

\section{Genetic Algorithm}

Genetic algorithm (GA) is inspired by a genetic and a natural selection. Implementation of GA for routing is surveyed in [15] showing that GA makes WSN lifetime longer. It is also surveyed in [16] in the case of energy efficient routing in WSN. From both surveys, although not specific to mobile agent, GA is a suitable algorithm for the mobile agent routing. 


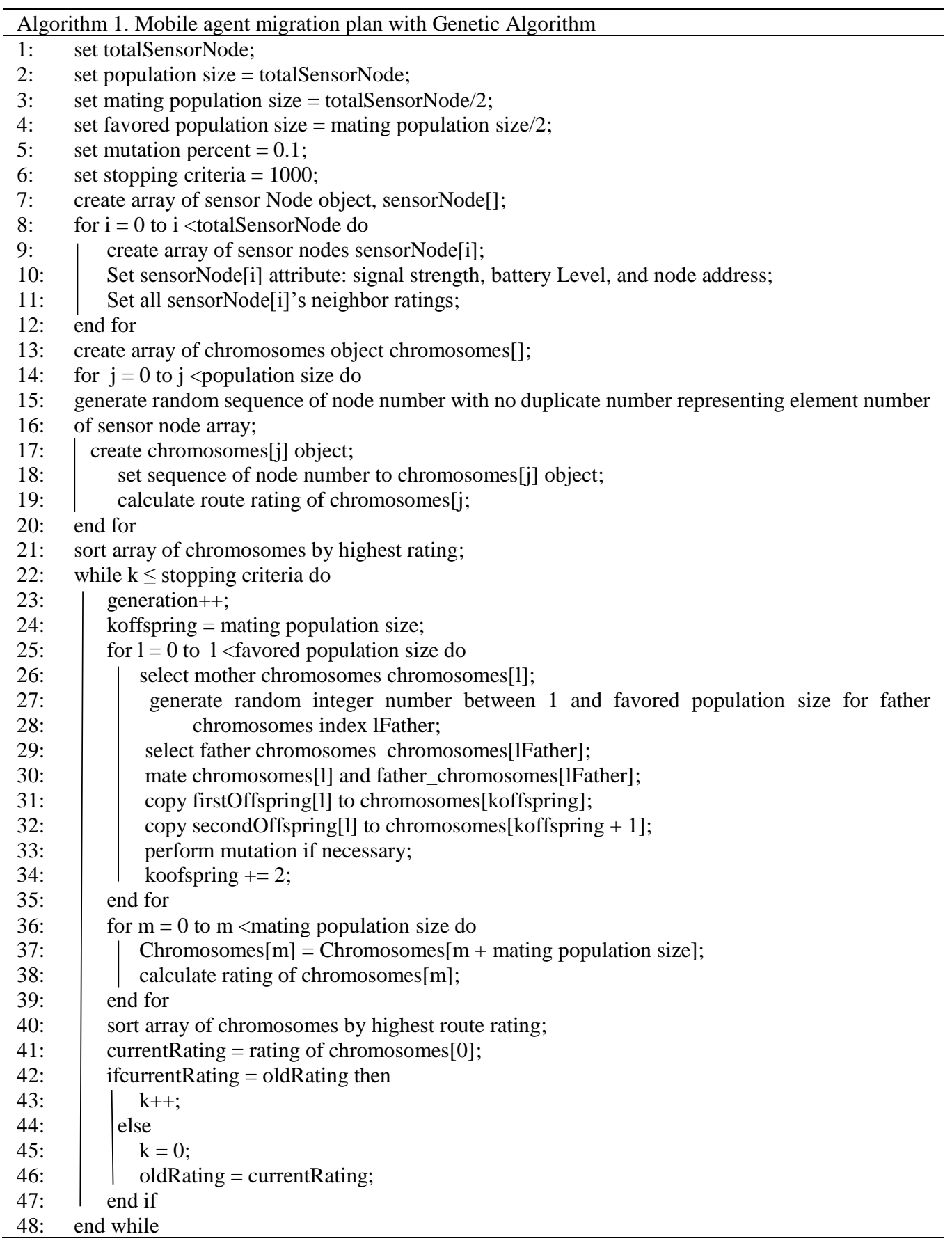

The proposed GA employment in WSN for mobile agent migration plan is shown in Algorithm 1. Here we modify GA to fit our WSN environment. Commonly, GA defines five procedures: initiation, generating initial population, calculating fitness, sorting chromosomes, and mutation. In initiation (line 8-12), we determine chromosomes. In this case, a chromosome represents a number sequence of sensor nodes generated randomly with no duplicate number (line 15-19). Total chromosome represents total population. The more population is set, the more opportunity to obtain good generations. Each mating process (line 25-33) will produce two offspring. When calculating fitness, we calculate total rating of route generated by each 
chromosome, and then sort the best chromosomes order by highest rating (line 39-40). The final step is mutation (Line 32) in which number sequence of sensor nodes in offspring chromosomes are exchanged. A random value is generated to choose whether to execute mutation, which will be executed if a random value is less than a defined value.

\section{Simulated Annealing}

Simulated Annealing (SA) is inspired by cooling process of steel. This technique mimics the behavior of steel, which is warmed up to a certain temperature and then cooled slowly. When a steel is heated to high temperature, the atoms of steel move freely and then move slowly when temperature level drops, which the composition of atoms will be more orderly (forming crystals) and be a minimum internal energy. The forming process of crystal depends on the rate of temperature reduction. If the rate of temperature decreases too fast, the steel may not reach the status of crystal and cause the damage in the material. Hence, it requires a slow cooling process to produce a good structure with small energy. This cooling process is called annealing. Here we modify the Boltzmann probability distribution to control temperature reduction. This probability is expressed by Equation 3.

$$
P(E)=e^{-\frac{R(p, e)}{k T}}
$$

If the generated random is less then $\mathrm{P}(\mathrm{E})$, and then annealing process is performed. It means that the swap process will be executed. Otherwise, the swap process will be not executed. The SA implemented for mobile agent migration plan is shown in Algorithm 2.

In the case of simulated annealing implementation for mobile agent migration plan, we need to perform iterations for every temperature level (line 14-35). If maximum iteration is reached, we have to reduce temperature level slowly. We also have to exchange the number sequence of sensor nodes, generated randomly (line $16-17$ ), that should be visited by mobile agent. This exchange process is called as swap (Line $21-24$ ). From this new number sequence of sensor nodes, we calculate its total rating (line $27-28$ ). The current sequence is put as the best mobile agent route (line 30) if the current total rating is more than previous rating. Then, we have to reduce temperature $\mathrm{T}$ (line 33 ) and continue iteration and annealing process (line $37-44$ ).

\section{Mobile Agent Migration and Data Preprocessing}

This section describes the next process after mobile migration plan is completed. After receiving a SENSE message from the head cluster agent, all sensor node agents do not only calculate the rating of neighbor sensor node, but also start to sense acceleration data of concrete beam vibration, transform it to frequency domain using FFT to find four dominant frequencies $F_{i}^{k}$ and its corresponding amplitude $\emptyset_{i}^{k}$ shown in Equation 4.

$$
F_{i}^{k}=\left[\begin{array}{cccc}
f_{1}^{1} & f_{2}^{1} & f_{3}^{1} & f_{4}^{1} \\
f_{1}^{2} & f_{2}^{2} & f_{3}^{2} & f_{4}^{2} \\
\ldots & \ldots & \ldots & \ldots \\
f_{1}^{N} & f_{2}^{N} & f_{3}^{N} & f_{4}^{N}
\end{array}\right], \quad \emptyset_{i}^{k}=\left[\begin{array}{cccc}
\varphi_{1}^{1} & \varphi_{2}^{1} & \varphi_{3}^{1} & \varphi_{4}^{1} \\
\varphi_{1}^{2} & \varphi_{2}^{2} & \varphi_{3}^{2} & \varphi_{4}^{2} \\
\ldots & \ldots & \ldots & \ldots \\
\varphi_{1}^{N} & \varphi_{2}^{N} & \varphi_{3}^{N} & \varphi_{4}^{N}
\end{array}\right]
$$

Here, $\mathrm{k}$ represents $\mathrm{k}^{\text {th }}$ sensor node and $\mathrm{i}$ represents $\mathrm{i}^{\text {th }}$ vibration frequencies. The sensor node agent then sends MA_REQ message to the head cluster agent for requesting the mobile agent. After receiving MA_REQ messages from all sensor nodes, the head cluster agent sends MA_DISPATCH message to the selected first sensor node to dispatch its mobile agent. The agent of the first sensor node then dispatches the mobile agent to migrate hop by hop. 


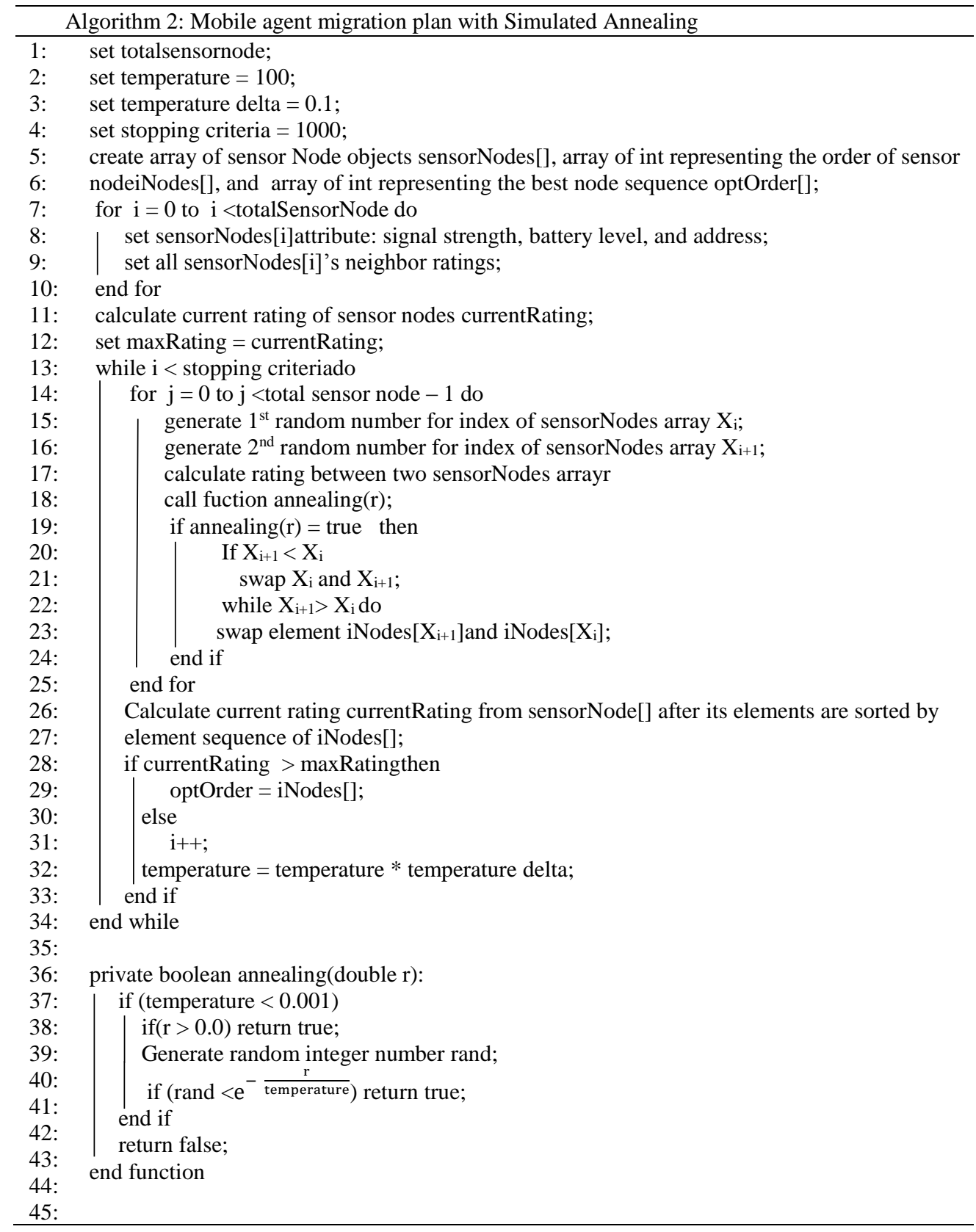

The cluster head agent, the sensor node agent, and the mobile agent consist of perceptors and actuators. A perceptor generates beliefs, and an actuator executes a specific intention. We implement agent programming language that defines logic formalisms called as belief and commitment described in [17] [18]. The agent anatomies are described in Table1.

Algorithm 3 shows how mobile agent performs in-network processing in WSN. At the first sensor node (line $10-15$ ), the mobile agent communicate with the sensor node agent to obtain $\mathrm{F}$ and $\varnothing$ and takes off from the node. It also brings a sequence of sensor node addresses that must be visited. When arriving in $\mathrm{k}^{\text {th }}$ sensor node (line $25-29$ ), the mobile agent also communicate to the $\mathrm{k}^{\text {th }}$ sensor node agent to collect $\mathrm{F}$ and $\varnothing$ and then takes off from the $\mathrm{k}^{\text {th }}$ node. Then, the data is loaded to mobile agent and aggregated with previous sensor node data. At the last sensor node 
(line 16 -23), the mobile agent performs data aggregation, calculation of average frequency of four dominant frequencies $\bar{F}_{i}$, and sends $\bar{F}_{i}$ and $\emptyset_{i}^{k}$ to a cluster head. Finally, the cluster head agent forwards these data to sink node.

Table 1. Agents Anatomy and Behavior

\begin{tabular}{|c|c|c|c|}
\hline Perceptor & Actuator & Belief & Intention \\
\hline \multirow{4}{*}{$\begin{array}{l}\text { SNPer } \\
\text { (Sensor Node Agent) }\end{array}$} & \multirow{2}{*}{ CommunicationAct } & Time to register node & Register my node \\
\hline & & Time to request mobile agent & Request mobile agent \\
\hline & FFTAct & Time to transform data & Do FFT \\
\hline & SensingAct & Time to Sense & Do sensing \\
\hline \multirow{5}{*}{$\begin{array}{l}\text { MAPer } \\
\text { (Mobile Agent) }\end{array}$} & MigrateActuator & Time to migrate to next node & Do migration \\
\hline & LandingAct & Landed on a node & Toggle a node \\
\hline & DataLoadingAct & Time to load data to MA & Load data to MA \\
\hline & DataProcessAct & Time to transmit data to $\mathbf{C H}$ & Consolidate data \\
\hline & SleepAct & Time to sleep & Torpor \\
\hline \multirow{3}{*}{$\begin{array}{l}\text { CHPer } \\
\text { (Cluster Head Agent) }\end{array}$} & CommunicationAct & Time to transceive packet & Transceive packet \\
\hline & MAMigrationPlanAct & Time to plan MA migration & Calculate optimal route \\
\hline & MADispatcherAct & Time to start MA migration & Execute MA migration \\
\hline
\end{tabular}

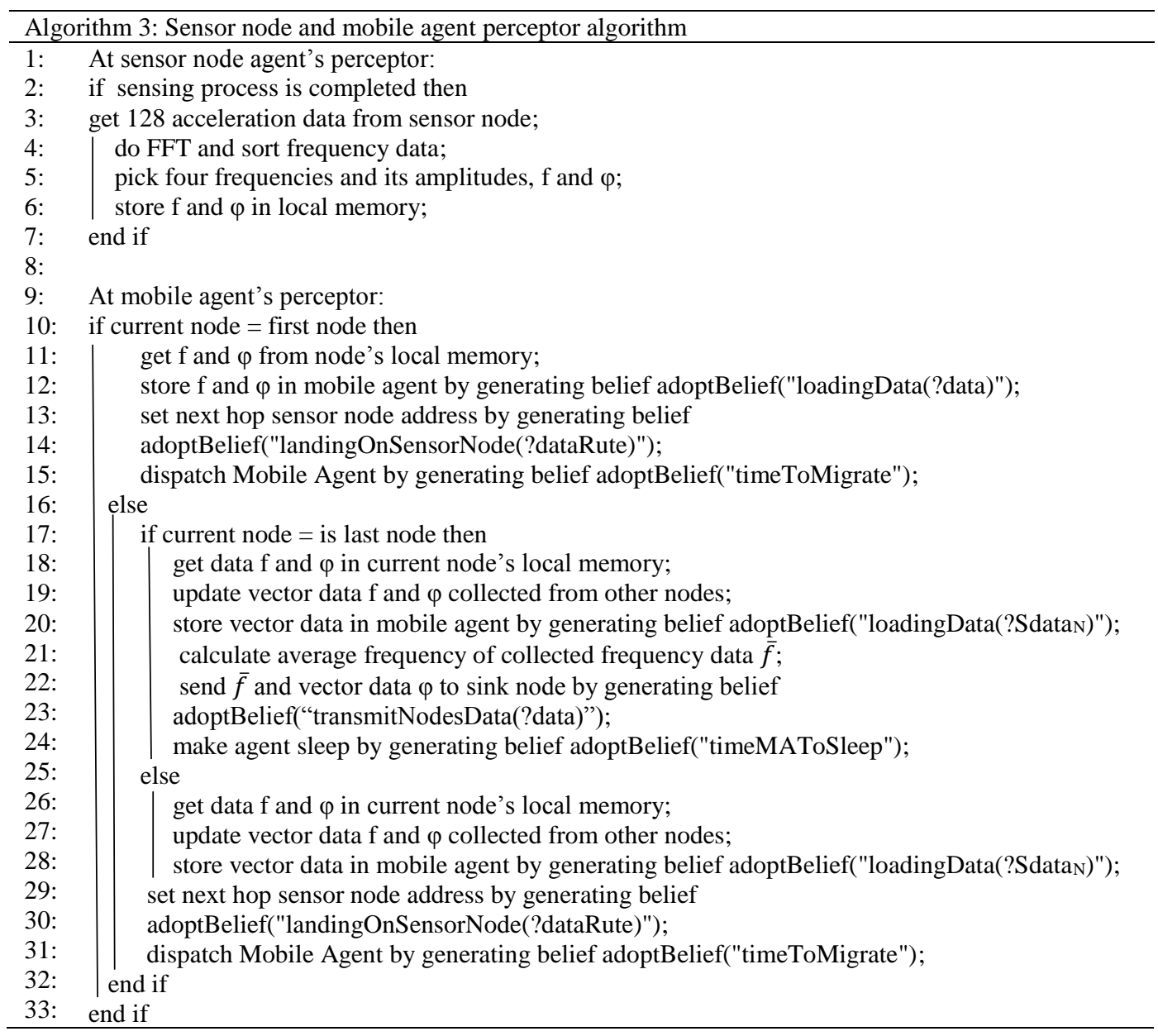




\section{Experimental Results}

Our propose method is tested in SunSPOT sensor nodes. The work related to implementation of SunSPOT can be found in [19]. SunSPOT contains an ARM920T core processor, 4 MB flash memory, and $512 \mathrm{~KB}$ RAM, $2.4 \mathrm{GHz} 802.15 .4$ radio transceiver, 3-axis accelerometer, a temperature, and a light sensor. SunSPOT Solarium Emulator is used for simulating our proposed in-network processing. Here, we simulated the cluster head agent that performs mobile agent migration plan and receives data from a mobile agent. The simulation environment is shown in Figure 3a. We simulate in-network processing in a different time step and total sensor node. Signal strength and battery level weight are set to $\mathrm{w}_{\mathrm{p}}=0.4$ and $\mathrm{w}_{\mathrm{e}}=0.6$ respectively.

We connect sensor nodes located in emulator to real five SunSPOTs. We employ a sensor node acting as a cluster head located in Solarium Emulator. The test result shows that our mobile agent can migrate hop by hop from first to last sensor node according to a set of routes that has been determined by the cluster head agent. A green LED is toggled when a mobile agent successfully arrives in a sensor node and performs data aggregation. Figure $3 \mathrm{~b}$ shows this experiment.

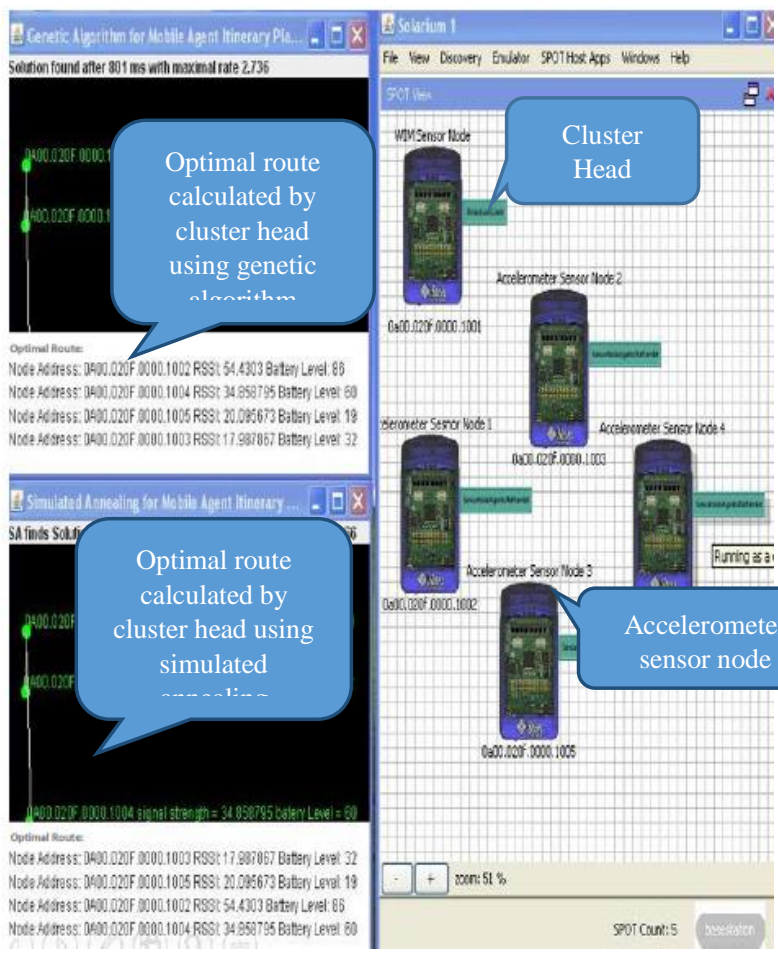

(a)

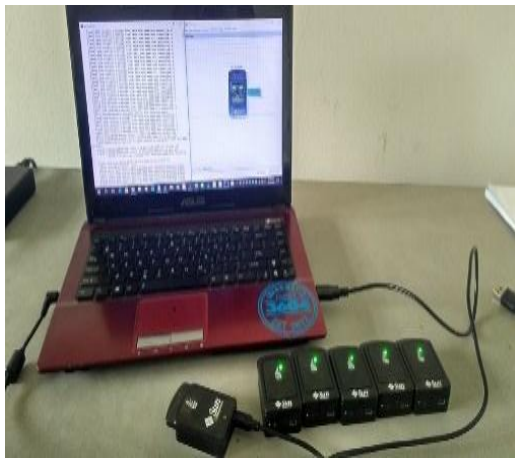

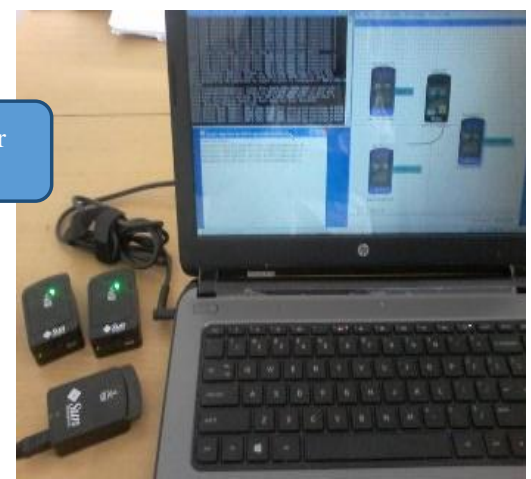

(b)

Figure 3. Simulation environment of in-network data processing in (a) SunSPOTs Solarium Emulator and in (b) real SunSPOTs sensor node.

\section{A. Case Study}

Acceleration data of concrete beam vibration is used as the case study. Here, we measure its first dominant frequency and corresponding amplitude using four sensor nodes. We give an impulse to a concrete beam to record its dynamic response. SunSPOT sensor node agents are programmed to sample data in $100 \mathrm{~Hz}$. Figure 4 shows this experiment. 

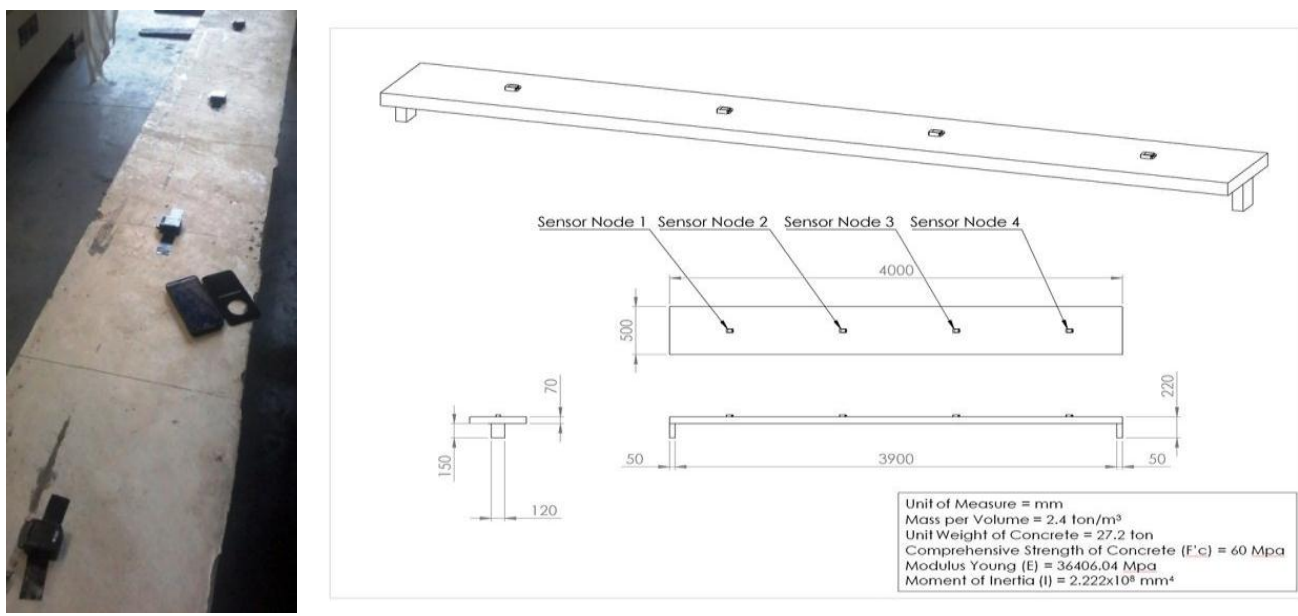

Figure 4. Experimental environment: (a) deploying four nodes on a concrete beam and

(b) property of concrete beam.

\section{B. Result Validation}

In this work, the first dominant frequencies and its corresponding amplitudes of concrete beam are identified as shown in Table II, which shows the average frequency $\bar{F}_{1}=22.07 \mathrm{~Hz}$. According to [20], the fundamental frequency of such concrete beam, idealized as single degree of freedom beam and simply-supported system, can be calculated using equation $f=$ $1.5078 \sqrt{\frac{E I}{m_{t} L^{3}}} x(0.826)$ where $\mathrm{E}$ is modulus young, $\mathrm{I}$ is moment of inertia, $m_{t}$ is total mass, and $\mathrm{L}$ is length of the beam. Using beam properties described in Figure $4 \mathrm{~b}$, we found first dominant frequency $F_{1}=25.16 \mathrm{~Hz}$. This value is close to the value calculated by the sensor node agent.

Table 2. First Frequency of Concrete Beam

\begin{tabular}{ccc}
\hline Sensor Node & Frequency $(\mathrm{Hz})$ & Amplitude x 9.8 $\mathrm{ms}^{-2}$ \\
\hline 1 & 22.656 & 0.0250 \\
\hline 2 & 21.875 & 0.0470 \\
\hline 3 & 21.875 & 0.0349 \\
\hline 4 & 21.875 & 0.0148 \\
\hline
\end{tabular}

\section{Performance Evaluation}

We measure the performance of two algorithms in term of the best solution that can be reached and its processing time. Here, we simulate 10 sensor nodes and a cluster head as shown in Figure 5a. Figure $5 \mathrm{~b}$ and $5 \mathrm{c}$ show comparison of these two algorithms for 10 sensor nodes or 362.880 possibilities of routes that must be searched by two algorithms. It can be seen that genetic algorithm is faster process than simulated annealing and in term of optimal solution simulated annealing is better.

In-network processing time and mobile agent migration time are defined in this work. First definition refers to duration time that is required for completing a round of in-network data processing including global network identification, mobile agent migration plan, and data preprocessing and mobile agent migration. Second definition refers to duration time required by a mobile agent to visit sensor nodes from the first to last node. Figure $5 \mathrm{~d}$ shows this simulation result, which describes in-network processing time under 30 seconds if a cluster contains 10 sensor nodes. 


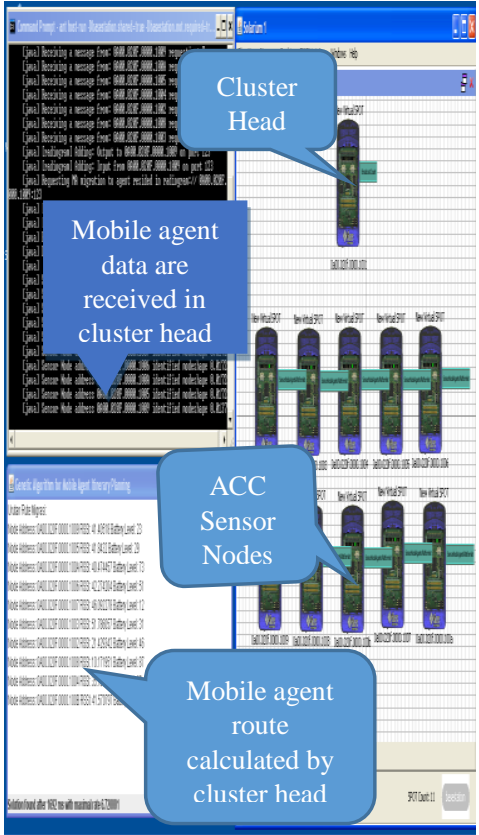

(a)

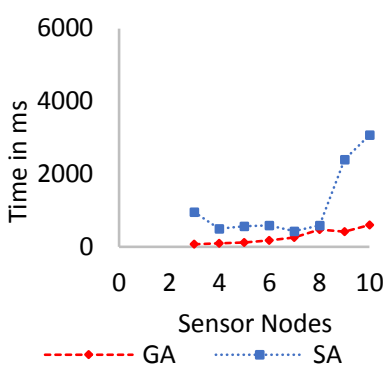

(b)

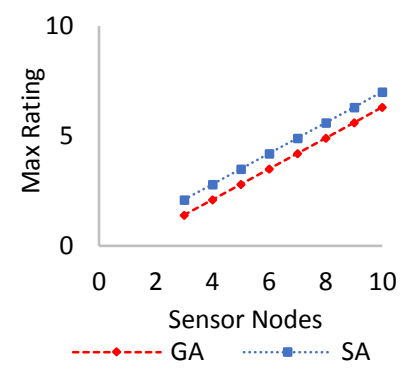

(c)

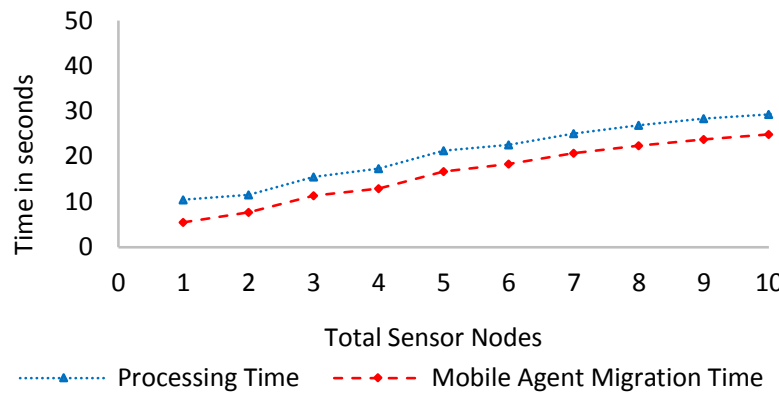

(d)

Figure 5 (a) Simulation of 10 sensor nodes and 1 cluster head, (b) Algorithm performance in term of processing time, (c) Algorithm performance in term of found best solution, and (d) innetwork processing time and mobile agent migration time.

\section{Method Comparison}

Energy consumption in each sensor node is measured to evaluate energy level reduction after performing a round of in-network processing. Mobile agent approach is compared with a traditional approach, called a client server approach. In a client-server approach, sensor nodes only deliver raw acceleration data to the cluster head. The comparison result is shown in Table III and energy reduction forour proposed and traditional approach is shown in Figure 6.

Table 3. Time Process and Energy Consumption of Two Different Approaches

\begin{tabular}{ccccc}
\hline \multirow{2}{*}{$\begin{array}{c}\text { Total } \\
\text { Node }\end{array}$} & $\begin{array}{c}\text { Mobile Agent } \\
\text { Approach (seconds) }\end{array}$ & $\begin{array}{c}\text { Traditional } \\
\text { Approach (seconds) }\end{array}$ & $\begin{array}{c}\text { Mobile Agent } \\
\text { Approach (\%) }\end{array}$ & $\begin{array}{c}\text { Traditional } \\
\text { Approach (\%) }\end{array}$ \\
\hline 3 & 14.2 & 33.7 & 0.14 & 1.85 \\
\hline 4 & 19.8 & 42.2 & 0.22 & 2.13 \\
\hline
\end{tabular}

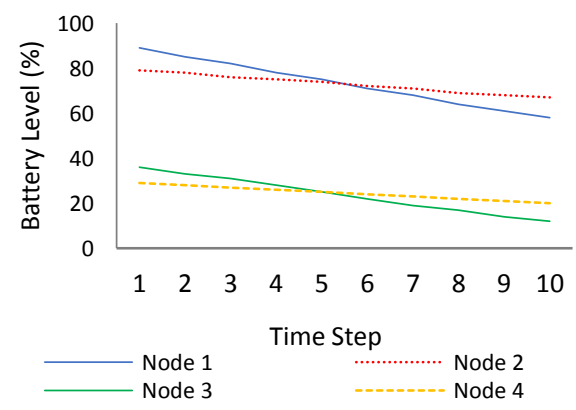

(a)

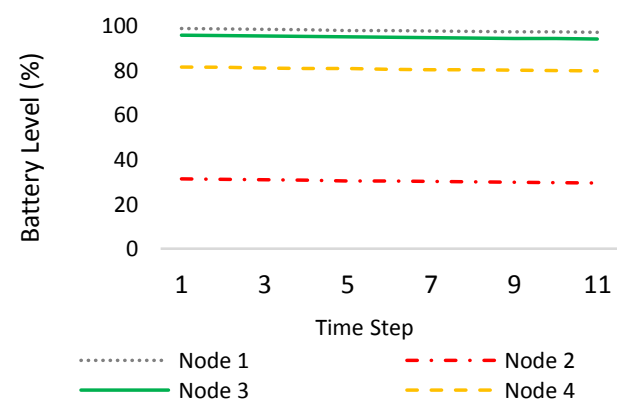

(b)

Figure 6. The energy reduction in (a) traditional approach and

(b) multiagent system in-network processing. 


\section{Conclusion}

This paper describes a work related to the concept of multiagent system in-network processing in WSN. We implement the combination architecture of one agent per sensor node and the mobile agent in real WSN, which considers aspects such as agent behavior and mobile agent migration plan. We have implemented BDI model in all involved agents and utilized genetic algorithm and simulated annealing for a mobile agent migration plan. When comparing multiagent system in-network processing with client server approach, we found that multiagent system approaches is superior to traditional approach. In addition, for the case of intelligent sensing for future works, our proposed multiagent system is recommended.

For future works, we propose multiagent system in WSN for specific application that implement above process. The candidate of application is bridge condition assessment and load rating using dynamic response. Here, we are going to develop the agent with capability to learn. In this case, we define the agent deployed in the observer sensor node that is able to make autonomous decisions and actions, probably based on Markov Decision Process, about when WSN should perform in-network processing.

\section{References}

[1] M. Chen, S. Gonzalez and V. C. M. Leung, "Application and Design Issues for Mobile Agent in Wireless Sensor Networks," IEEE Wireless Communication, vol. 14, no. 6, pp. 20-26, 2007.

[2] R. Tynan, G. M. O'Hare, D. Marsh and D. O'Kane, "Multi-agent System Architectures for Wireless Sensor Networks," in 5th International Conference, Atlanta, GA, USA, 2005.

[3] K. H. S. Hla, Y. Choi and J. S. Park, "The Multi Agent System Solutions for Wireless Sensor Network Applications," in KES International Symposium on Agent and MultiAgent Systems: Technologies and Applications, Incheon, Korea, 2008.

[4] A. Montoya, D. C. Restrepo and D. A. Ovalle, "Artificial Intelligence for Wireless Sensor Network Enhancement," Smart Wireless Sensor Networks, pp. 73-81, 2010.

[5] P. Gil, A. Santos and A. C, "Dealing with Outliers in Wireless Sensor Networks: an Oil Refinery Application," IEEE Transactions on Control Systems Technology, vol. 22, no. 4, pp. 1589 - 1596, 2014.

[6] C. K, A. Mpitziopoulos, D. G and G. Pantziou, "Effective Determination of Mobile Agent Itineraries for Data Aggregation on Sensor Networks," IEEE Transactions on Knowledge and Data Engineering, vol. 22, no. 22, pp. 1679-1693, 2010.

[7] X. Wang, M. Chen, T. Kwon and H. Chao, "Multiple mobile agents' itinerary planning in wireless sensor networks: survey and evaluation," IET Communications, vol. 5, no. 12, p. 1769-1776, 2011.

[8] M. Chen, L. T. Yang, T. Kwon, L. Zhaou and M. Jo, "Itinerary Planning for EnergyEfficient Agent Communication in Wireless Sensor Network," IEEE Transactions on Vehicular Technology, vol. 60, no. 7, pp. 3290 - 3299, 2011.

[9] M. Sharawi, I. A. Saroit, H. El-Mahdy and E. Emary, "Routing Wireless Sensor Networks Based On Soft Computing Paradigms: Survey," International Journal on Soft Computing, Artificial Intelligence and Applications (IJSCAIS), vol. 2, no. 4, pp. 21-36, 2013.

[10] G. I. Simari and S. D. Parsons, "Bridging the Gap for Autonomous Agents," in Markov Decision Processes and the Belief-Desire-Intention Model, New York, Springer-Verlag, 2011, p. 63.

[11] Q. Sun, W. Yu, N. Kochurov, Q. Hao and F. Hu, "A Multi-Agent-Based Intelligent Sensor and Actuator Network Design for Smart House and Home Automation," Journal of Sensor and Actuator Networks, vol. 2, pp. 557-588, 2013. 
[12] K. Shah, M. D. Fransesco and M. Kumar, "Distributed Resource Management in Wireless Sensor Network Using Reinforcement Learning," Wireless Network, vol. 19, no. 5, p. 705724, 2012.

[13] A. Sardouk, R. Rahim-Amoud, L. Marghem-Boulahia and D. Gaiti, "Agent Startegy Data Gatheringfor Long Life WSN," International Journal of Computer Networks and Communications (IJCNC), vol. 2, no. 5, pp. 71-90, 2010.

[14] T. Nanayakkara, M. N. Halgamuge, P. Sridhar and A. M. Madni, "Intelligent Sensing in Dynamic Environments Using Markov Decision Process," Sensors, vol. 11, pp. 12291242, 2011.

[15] R. V. Kulkarni, F. Anna and G. K. Venayagamoorthy, "Computational Intelligence in Wireless Sensor Networks: A Survey," IEEE Communications Surveys \& Tutorials, vol. 13, no. 1, pp. 68-96, 2011.

[16] M. A. Adnan, M. A. Razzaque, I. Ahmed and I. F. Isnin, "Bio-Mimic Optimization Strategies in Wireless Sensor Network: A Survey," Sensors, vol. 14, pp. 299-345, 2014.

[17] F. Aiello, A. Carbone, G. Fortino and S. Galzarano, "Java-Based Mobile Agent Platform for Wireless Network Sensor," in The International Multiconference on Computer Science and Information Technology, 2010., 2010.

[18] R. Lopes and F. Assis, "MASPOT: A Mobile Agent System for SunSPOT," in Tenth International Symposium on Autonomous Decentralized Systems, Tokyo and Hiroshima, 2011.

[19] H.-d. Shih, X. Zhang, D. S. Wei, K. Naik and R.-C. Chen, "Design and Implementation of Mobile Sensor Network Testbed Using SunSPOTs," International Journal of Future Computer and Communication, Vol. 2, No. 2, 2013., vol. 2, no. 2, pp. 115-120, 2013.

[20] A. A. Islam, H. Hamid, A. Jaroo and F. Li, "Bridge Condition Assessment and Load Rating using Dynamic Response: Technical Report for The Ohio Department of Transportation Office of Statewide Planning Research," Younstown State University, Columbus, Ohio, 2014.

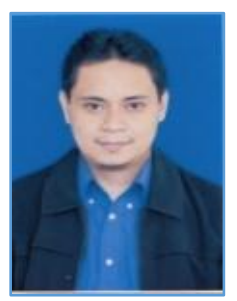

Seno Adi Putra received graduate degree in physics science from Department of Physics in Bandung Institute of technology and master degree in electrical engineering from School of Electrical Engineering and Informatics in Bandung Institute of Technology. Currently, He is a Ph.D student in the same institution as his master degree. His Research interests include wireless sensor network, multiagent system, intelligent system, and machine learning.

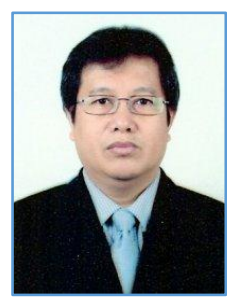

Bambang Riyanto Trilaksono received the graduate degree from Electrical Engineering in Bandung Institute of Technology, master degree from Electrical Engineering, Waseda University, Japan, and $\mathrm{PhD}$ degree from the same institution as master degree. Currently, he is a professor in Control and Computer System Research Group in Bandung Institute of Technology. His research interests include robust control, intelligent control \& intelligent systems, discrete event systems, control applications, robotics, and embedded control systems. 


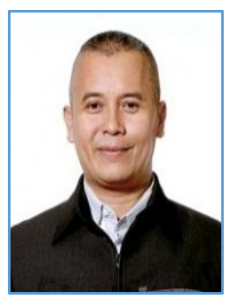

Agung Harsoyo received the graduate degree from Electrical Engineering in Bandung Institute of Technology, master degree from Telecommunication Engineering in ENST-B, and $\mathrm{PhD}$ degree from telecommunication engineering in UBS. Currently he is a researcher in Control and Computer System Research Group at Bandung Institute of Technology. His research interests include wireless sensor network, control and computer system, and intelligent system.

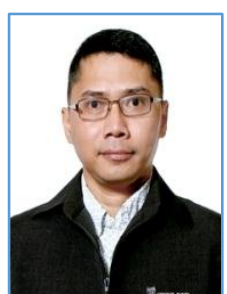

Achmad Imam Kistijantoro received graduate degree from Department of Informatics in Bandung Institute of Technology, master degree from Department of Informatics in TU Delft, Netherlands, and PhD degree from Computing Department at University of Newcastle upon Tyne, United Kingdom. He is a researcher in Distributed and Parallel Computing Research Group at Bandung Institute of Technology. His research interests includes distributed system, parallel computation, and high performance computation 